\title{
Utilisation of earthworm meal in partial replacement of soybean and fish meals in diets of broilers
}

\begin{abstract}
To study the effect of worm meal (WM) as partial replacement of soybean and fish meals in the diets, a total of 245 day-old broiler chicks were randomly assigned to five different treatment groups $(0 \%, 5 \%, 10 \%, 15 \%$ and $20 \% \mathrm{WM})$ in partial replacement of soybean and fish meals for a period of 6 weeks. The final body weight, growth rate and feed efficiency of the $10 \%$ and $15 \% \mathrm{WM}$ groups broiler were better $(\mathrm{P}<0.05)$ than that of the control group with no effect on feed intake. The digestibility of crude protein for WM was 63\%. 10\% WM group gave a higher $(\mathrm{P}<0.05)$ lactic acid bacteria counts and showed no significant difference $(\mathrm{P}>0.05)$ in Enterobacteriaceae count or fecal $\mathrm{pH}$. These results suggest that WM could be used to replace soybean and fish meals between 10 to $15 \%$ in broiler diets.
\end{abstract}

Keyword: Earthworm; Worm meal; Soybean meal; Fish meal; Broiler 